\title{
The Effects of Dispersal Gradient and Pathogen Life Cycle Components on Epidemic Velocity in Computer Simulations
}

\author{
Kathryn E. Sackett and Christopher C. Mundt
}

Botany and Plant Pathology Department, 2082 Cordley Hall, Oregon State University, Corvallis 97331. Accepted for publication 30 April 2005.

\begin{abstract}
Sackett, K. E., and Mundt, C. C. 2005. The effects of dispersal gradient and pathogen life cycle components on epidemic velocity in computer simulations. Phytopathology 95:992-1000.

The velocity of expansion of focal epidemics was studied using an updated version of the simulation model EPIMUL, with model parameters relevant to wheat stripe rust. The modified power law, the exponential model, and Lambert's general model were fit to primary disease gradient data from an artificially initiated field epidemic of stripe rust and employed to describe dispersal in simulations. The exponential model, which fit the field data poorly $\left(R^{2}=0.728\right.$ to 0.776$)$, yielded an epidemic

model fit the field data well $\left(R^{2}=0.962\right.$ to 0.988$)$ and resulted in dispersive epidemic waves (velocities increased over time for the entire course of the epidemic). The field epidemic also expanded as a dispersive wave. Using parameters based on the field epidemic and modified power law dispersal as a baseline, life cycle components of the pathogen (lesion growth rate, latent period, infectious period, and multiplication rate) and dispersal gradient steepness were varied within biologically reasonable ranges for this disease to test their effect on dispersive wave epidemics. All components but the infectious period had a strong influence on epidemic velocity, but none changed the general pattern of velocity increasing over time.
\end{abstract} that expanded as a traveling wave (i.e., at a constant velocity), after an initial buildup period. Both the modified power law and the Lambert

One of the fundamental challenges of plant pathology is to understand the spread of plant diseases in time and space. Focal epidemics, in particular, have drawn considerable interest $(62,65)$. These are epidemics in which disease spreads outward over time from the initial site of infection. Diseases such as potato late blight (caused by Phytophthora infestans) and wheat stripe rust (caused by Puccinia striiformis), among others, exhibit focal spread in the field (67). To quantify this type of disease spread, Berger and Luke (8) introduced the concept of isopathic velocity. An isopath is a contour in space of constant disease level. As a focal epidemic progresses, isopaths expand outward from the center of the focus. The rate of movement of an isopath in a particular direction is its velocity, expressed in units of distance per time (e.g., meters/day).

Understanding how fast epidemics spread in space has important implications for controlling disease, because fast-moving epidemics may be far more challenging to combat than slow-moving ones. In addition, since dispersal is a universal phenomenon in ecology, work on the spread of plant pathogens may inform study of a variety of other systems of practical interest, including spread of arthropod pests and weeds (23), spread of invasive animal species (2), restoration and conservation of natural vegetation (23), geographic movement of genetic traits in a population (49), deployment of biocontrol organisms $(19,23,65,67)$, and the potential dispersal of genetically modified organisms (67).

Research on the expansion of plant disease foci in the field is expensive both in terms of labor and the amount of land required (35), and field data on epidemic velocity are limited (1-3,6,8, $10,20,23,34,38,44,57)$. Typically, the spatial extent of experimen-

Corresponding author: K. E. Sackett

E-mail address: sackettk@science.oregonstate.edu

DOI: 10.1094/PHYTO-95-0992

(C) 2005 The American Phytopathological Society
Additional keywords: Puccinia striiformis, Triticum aestivum. tal plots restricts the ability of researchers to characterize velocity over an extended period of time, because isopaths quickly move beyond the borders of small plots. Analytic and simulation-based approaches have yielded some insight, but have also given rise to a controversy that field experiments have yet to resolve (65). The disagreement lies in whether plant epidemics expand at a constant velocity, after a brief buildup period, or whether velocity is unbounded and increases over time. Epidemics with constant velocity are said to expand as traveling waves, with disease gradients (plots of disease level versus distance from the source) that maintain a constant shape after the buildup period is past. Dispersive waves, on the other hand, are exhibited by epidemics with increasing velocity; disease gradients become shallower as the epidemic progresses (15).

Analytic studies have resulted in conflicting conclusions regarding this controversy. While the models developed by Minogue and Fry $(37)$ and van den Bosch et al. $(58,59)$ predicted a constant velocity, Ferrandino (15) found that velocity should increase with time. All three models represent fungal diseases that are spread via airborne spores. The important difference appears to be in the assumptions regarding the dispersal of those spores. In order to incorporate a spatial aspect into disease models, it is necessary to make assumptions about the distribution of propagules around a source plant. This dispersal gradient (or, equivalently, contact distribution) may be derived from physical principles such as turbulent diffusion $(15,18,58)$ or may be empirical, based on experimental observations of spore dispersal $(14,17)$.

Mollison (39) showed that traveling waves are expected only if the tail of the dispersal gradient is exponentially bound, while dispersal gradients with non-exponentially bound tails produce dispersive waves. Indeed, the physical assumptions of Minogue and Fry (37) and van den Bosch et al. (59), though different, resulted in exponentially bound dispersal gradients-the double geometric and Bessell distributions, respectively-and their models predicted traveling waves. In contrast, Ferrandino's dispersal gradient was not exponentially bound and resulted in a disper- 
sive wave (15). Simulation studies of epidemic velocity show the same connection between dispersal gradient and velocity $(14,50$, 66,68).

The issue, it seems, would be simple enough to resolve if the "true" nature of the tails of dispersal gradients (i.e., exponentially bound or not) could be determined. The nature of the tails of the dispersal gradient may be different for different plant-pathogen systems, depending on such factors as the mechanism of dispersal, crop structure, and local environmental conditions (18). Fitt et al. (17) suggested that dispersal gradients of splash-dispersed droplets were fit better by the exponential model, while those for fungi with small $(<10 \mu \mathrm{m})$ airborne spores were fit better by the power law, which is not exponentially bound in the tail. There is, however, no definitive rule for predicting the character of the dispersal gradient, and indeed it is often impossible to determine from a given data set whether or not the gradient tail is exponentially bound $(16,17,20)$. One difficulty is that exponentially bound functions (such as the exponential, equation 1 below) and nonexponentially bound functions (such as the modified power law, equation 2 below) can take on similar values, particularly near the source where data are easiest to come by. Dispersal data collected at relatively large distances from the source, therefore, are crucial for characterizing a dispersal gradient and have the potential to shed light on the behavior of epidemic velocity as well.

Although plant-pathogen dispersal gradients are critical to the behavior of expanding epidemics, previous studies of epidemic velocity $(15,37)$ have often relied on physical theories of spore dispersal, rather than field data. An exception is the work of van den Bosch et al. $(57,59)$, who fit the primary disease gradient data of wheat stripe rust and spinach downy mildew (caused by Peronospora farinosa) to an exponential model. The field data extended less than $2 \mathrm{~m}$ from the inoculum source, however, and thus did not account for the influence of longer distance dispersal on epidemic waves. Frantzen and van den Bosch (20) also collected primary disease gradient data, this time with the specific goal of predicting whether an epidemic of Puccinia lagenophorae on Senecio vulgaris would expand as a dispersive or traveling wave. The extent of their data was also limited $(1 \mathrm{~m}$ from the source), and they were unable to distinguish whether or not the gradient was exponentially bound. Disease data over time, however, suggested a dispersive wave. More work is needed to tie analytical and simulation results to real plant disease epidemics.

In terms of disease management, dispersal gradients cannot generally be manipulated to control disease spread. Other factors that may influence velocity are characteristics of the pathogen's life cycle, which may in turn be influenced by environmental conditions, host vigor and genetic resistance, pathogen virulence, and pesticide application. Berger and Luke (8) found that resistance in oats to crown rust was associated with reduced epidemic velocity. Jeger et al. (24) observed reduced spread of Stagonospora nodorum on wheat in mixtures of susceptible and resistant plants, relative to pure stands of the susceptible variety. On the other hand, Minogue and Fry's field study (38) detected no difference in the rate of spread of potato late blight among plots of susceptible plants, moderately resistant plants, and susceptible plants with a fungicide applied.

Components of a pathogen's life cycle that may affect the progress of plant epidemics include sporulation rate, infection efficiency, length of latent period, length of infectious period, and rate of lesion growth $(7,42)$. There is considerable interest in quantifying these components for a variety of pathosystems in order to assess host resistance $(11,12,53,56)$, select pathogen strains for biological control of weeds (31), and evaluate the effect of cultural practices on disease (46). The interaction of these components over multiple generations is complicated, however, and analytic methods and simulations have been used to integrate their effects into succinct measures of disease, such as rate of disease increase $(r)$, area under the disease progress curve (AUDPC), severity at a given date $(29,33,43,47,52,54,61)$, and epidemic velocity $(15,37,59)$. In studies where velocity has been examined, none has incorporated lesion growth as a disease component. For diseases with indeterminate lesion size, such as wheat stripe rust and potato late blight, the rate of lesion growth can affect disease progress significantly $(7,28)$.

In the present study, we used a spatially explicit simulation model to examine the influence of dispersal gradient and pathogen life cycle parameters on disease spread. Dispersal in the model was based on field data extending as far as $79 \mathrm{~m}$ from the source (45). Spatiotemporal field data was also available over the course of the epidemic, allowing corroboration of simulation results (10). Three different dispersal models were then fit to the gradient data and used in simulations to determine how the velocity of epidemic spread is influenced by the tail shape of the dispersal gradient. We were able to establish that an exponentially unbound function both fit the dispersal data and produced a simulated epidemic with the dispersive wave characteristics of the field epidemic. This in turn allowed us to explore the effects of variation in pathogen life cycle components, including lesion expansion, on dispersive epidemic waves. All previous such studies $(37,58,59)$ have been limited to traveling waves.

\section{MATERIALS AND METHODS}

Simulation model. The computer simulator EPIMUL was first introduced by Kampmeijer and Zadoks (25) to address the effectiveness of host mixtures and mosaics in controlling disease. The version of EPIMUL used in this study is essentially the same as the original, with a few enhancements (power law dispersal [41] and lesion growth [28], plus asymmetrical dispersal and exponential or Lambert model dispersal as described below).

EPIMUL divides the host plant population into a two-dimensional spatial grid in which each cell of the grid may represent an individual plant, a group of plants, or a field, depending on the application. The user may specify the arrangement in the grid of hosts of varying degrees of susceptibility to a pathogen, as well as the location and quantity of pathogen inoculum that initiates the epidemic. Each cell of the grid contains a number of potential infection sites, representing unit lesions, each of which exists in one of four states: healthy, latent, sporulating, or removed.

Pathogen propagules (spores) are dispersed daily among the cells according to one of three dispersal models: the modified power law model (40),

$$
y=a(x+c)^{b}
$$

the exponential (26),

$$
y=a \exp (-b x)
$$

or the more general model proposed by Lambert et al. (27),

$$
y=a \exp \left(-b x^{n}\right)
$$

which is capable of describing curve shapes between those of the first two models. In each equation above, $y$ is the number of effective spores dispersed to a distance $x$ from the source lesion. The parameter $a$ is proportional to the amount of inoculum produced at a source location; $b$ influences the steepness of the gradient; $c$ is an offset parameter that allows the power law to have a finite value at $x=0$; and $n$ is the shape parameter of the Lambert model. (Note, however, that when performing regressions to fit the modified power and Lambert models to data, the parameters are strongly correlated, so interpretation of their values should be approached cautiously.) In each equation, $a$ has no effect on the shape of the gradient, but is used to determine the proportionality between upwind and downwind gradients. EPIMUL normalizes the combined upwind and downwind gradients before using them for dispersal, so the form of the gradient model does not affect the quantity of spores dispersed from each lesion. 
After dispersal, new infections are initiated in proportion to the number of uninfected sites in the destination cell. New lesions begin producing spores after a fixed latent period and continue to do so until the infectious period is complete, after which the site is considered removed-neither infectable nor infectious. While infectious, lesions grow at a rate proportional to the number of healthy sites in the cell. Plant tissue that becomes infected via lesion growth does not pass through a latent period before beginning to produce spores. The rate of pathogen reproduction is the daily multiplication factor (DMFR): the number of daughter lesions per parent lesion per day that would be produced in an unlimited, completely healthy and susceptible population. This parameter incorporates spore production, infection efficiency, and loss of spores to the ground and atmosphere. It can therefore be thought of as the number of "effective spores" to originate from a lesion each day.

EPIMUL was modified for this study from previous versions in order to (i) allow a grid as large as $105 \times 105$ cells; (ii) produce an additional output data file to speed calculations of isopath velocities; (iii) include the exponential and Lambert models as options for dispersal gradients, in addition to the modified power law model already available (41); and (iv) allow the input of both upwind and downwind gradients to simulate the asymmetrical dispersal typical of directional wind patterns. Dispersal in other directions is modeled as a smooth transition between downwind and upwind gradients.

A baseline simulation was created using archival and field data to mirror an actual wheat stripe rust epidemic that took place as part of a field experiment. Each of six factors (dispersal gradient model, steepness of dispersal gradient, latent period, infectious period, lesion growth rate, and reproduction rate) was then varied one at a time to determine its effect on the velocity of expansion of the focal epidemic.

Field experiment. The baseline simulated epidemic was constructed to mimic a field experiment conducted near Hermiston, OR, during the spring of 2002. This experiment was chosen from four possible experiments (two locations, 2 years) for the precision and extent of the dispersal data available (45). A full description of the experiment is given by Cowger et al. (10).

Winter wheat cv. Jacmar, which is highly susceptible to $P$. striiformis, was planted in three 6.1-m-wide field plots. The lengths of the plots were 131, 146, and $171 \mathrm{~m}$. Plots were oriented approximately parallel to the prevailing winds and were separated from one another and other inoculated plots by $16.8-\mathrm{m}$ swaths of resistant winter wheat cv. Stephens. The wheat was planted in October 2001. In March 2002, a square area in each plot measuring 1.52 by $1.52 \mathrm{~m}$, centered in the crosswind direction and shifted upwind of center in the other direction, was inoculated using urediniospores of $P$. striiformis race CDL 5 (CDL $=$ USDAARS Cereal Disease Laboratory, Pullman, WA). No wheat cultivars susceptible to $P$. striiformis were commonly grown in the area at the time this study was conducted, and risk of contamination of field plots by indigenous populations of the pathogen was considered low.

Disease severity (percentage of leaf area visibly affected by stripe rust) was assessed weekly in the three plots at $6.1-\mathrm{m}$ or closer intervals upwind and downwind from the focus (the loca- tion of the inoculation) until the plants began to senesce naturally at the beginning of June. An additional, more intensive round of data collection took place after sporulating lesions began to appear outside the focus but before a third generation of the pathogen was evident (45). To get a precise estimate of the shape of this primary disease gradient, disease assessments were made at 39 locations in each of the two longest plots, including the focus. (Gradient assessment was not possible in the third plot due to planting irregularities.) At each location, the assessment was made on the F-3 leaf (the third leaf beneath the flag leaf) of each tiller in a $0.3-\mathrm{m}$ section of each of two rows $0.9 \mathrm{~m}$ apart, one on each side of the long axis of the plot. The assessment consisted of either a count of the total lesions or a visual assessment of percent severity (leaf area covered), and was later converted to an estimate of the number of lesions per leaf (45). Values in the focus were transformed using a multiple infection transformation (21) to remove the effect of the first generation of disease, which took place only in the focus (45). The resulting primary disease gradient was taken to be proportional to the dispersal gradient.

Baseline simulation. Plot layout. The same plot layout was used for all simulations and was constructed to correspond as closely as possible to the conditions of the field experiment. One cell in the EPIMUL grid represented an area the same size as the inoculated area in the field experiment ( 1.52 by $1.52 \mathrm{~m}$ ). Since the shape of the dispersal gradient may change with the size and shape of the inoculum source (22), the dispersal data collected in the field are directly applicable only to a source the same size and shape. The simulated plot was three rows wide and 89 columns long, with the inoculated focus placed in the second row of the 24th column. This width was slightly narrower than the field plot but preserved the position of the focus in the center of the plot.

A number of different calculations were made to determine an appropriate number of potential infection sites per cell. Estimates derived both from field observations and from the literature came to approximately 500,000 infection sites per cell. An initial disease level of 115,000 lesions in the focus cell was calculated using the first-generation disease severity observed in the focus in the field $(23 \%$ severity $\times 500,000$ infection sites). Since data were gathered weekly for the field experiment, severity data were also extracted from EPIMUL at 7-day intervals.

Dispersal gradients. Estimates of gradient model parameters were derived from nonlinear least squares regression on the logtransformed response, using log-transformed versions of the models: $\ln y=\ln a-b x$ for the exponential, $\ln y=\ln a-b \ln (x+c)$ for the modified power law, and $\ln y=\ln a-b x^{n}$ for the Lambert. Locations in the field where no lesions were found in either replicate were excluded from the analysis since the logarithm is not defined at zero. Data points beyond these zeroes where lesions were found were also excluded in order not to introduce bias into those regions. (This was the remedy for zero readings preferred by Minogue [35].) Nonzero values extended from the focus to $79.2 \mathrm{~m}$ downwind and $12.2 \mathrm{~m}$ upwind. The modified power law gave a superior fit for both the upwind and downwind gradients, when evaluated using residual plots and $R^{2}$ values (45) (Table 1 , Fig. 1), and therefore was used for the baseline simulation. The modified power law also provided a good fit for three other data sets (six gradients) from similar experiments (45).

TABLE 1. Dispersal gradient model parameters estimated from Hermiston, OR, 2002 primary disease gradient data of wheat stripe rust (45) ${ }^{\mathrm{a}}$

\begin{tabular}{|c|c|c|c|c|c|c|c|c|}
\hline \multirow[b]{2}{*}{ Gradient model } & \multicolumn{4}{|c|}{ Downwind } & \multicolumn{4}{|c|}{ Upwind } \\
\hline & $a$ & $b$ & $c$ or $n$ & $R^{2}$ & $a$ & $b$ & $c$ or $n$ & $R^{2}$ \\
\hline Modified power law & 431.8 & 2.29 & 0.848 & 0.987 & 6.912 & 2.42 & 0.180 & 0.988 \\
\hline Exponential & 18.59 & 0.106 & $\ldots$ & 0.776 & 10.91 & 0.658 & $\ldots$ & 0.728 \\
\hline Lambert & 895.9 & 2.85 & 0.312 & 0.962 & 452.0 & 4.84 & 0.310 & 0.987 \\
\hline
\end{tabular}

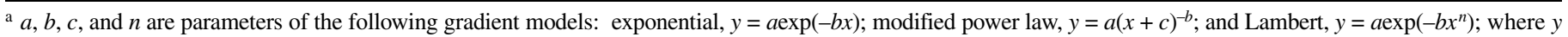
is the number of lesions per leaf at a distance $x$ (in meters) from the center of the inoculated focus. 
Latent period. Temperature is the main factor influencing latent period (the time between infection and first production of the next generation of spores) for stripe rust on wheat. Based on the mean daily temperature at the Hermiston field station during the spring of $2002,11.7^{\circ} \mathrm{C}$, the average latent period was estimated to be 17 days $(48,55,63)$. Also, in a growth chamber experiment using a diurnal temperature regime similar to that observed in the field at Hermiston during the spring of 2002 and using the same wheat cultivar and pathogen isolate as in the field experiment, the average latent period was 17 days (data not shown).

Infectious period. Luo and Zeng (32) reported wheat stripe rust infectious periods ranging from 6.05 to 7.39 days in four slowrusting varieties and 11.61 days in a susceptible variety. Data given by van den Bosch et al. (57) showed that the bulk of stripe rust spores were produced in the 14-day period starting 6 days after inoculation. The most conservative choice of infectious period for a susceptible cultivar would be the largest of these values, and so 14 days was used for the baseline simulation.

Daily multiplication factor. This parameter is the most difficult to estimate. Since it is a measure of effective spores, determining its value directly would mean counting all the daughter lesions produced by a single lesion in a healthy, susceptible population of infinite extent. In the present case, it would also require an accurate count of the total number of parent lesions in the inoculated focus before dispersal. Instead, an indirect approach was used. The isolated effect of the DMFR is most evident when the first dispersed generation of the pathogen appears outside the inoculated focus. At this time, there is no overlap of generations outside the focus, and thus no reduction of infection efficiency due to already infected tissue. Also, growth of lesions beyond their initial size has not yet begun outside the focus. Trial simulations with various DMFRs were run, and the disease gradient at this time was compared with the field epidemic. Gradients were most similar when the DMFR was set at five daughter lesions per parent lesion per day.

Lesion growth. Field estimates of the lesion growth rate of P. striiformis range from 15 to $76 \%$ per day $(13,28,33)$. In preliminary trial simulations, a rate of $25 \%$ per day produced a simulated epidemic more similar to the field epidemic than larger or smaller values. This value was used for the baseline simulation.

Simulations. In order to evaluate the importance of assumptions about the shape of the dispersal gradient, the exponential, modified power law, and Lambert models were each used to represent the gradients, while keeping all other model parameters at their baseline values. Dispersal model parameters were estimated based on the field experiment, as described above (Table 1). Upwind and downwind data were fit separately.

To test the effect of the steepness of the dispersal gradient on epidemic velocity, the exponent, $b$, of the modified power law was varied, with $a$ and $c$ held constant. For simplicity, the same gradient was used to model dispersal in both the upwind and downwind directions in this round of simulations. The offset parameter, $c$, was kept at the baseline downwind value of $0.848 \mathrm{~m}$. Using downwind gradients for both upwind and downwind dispersal slows disease spread slightly, but does not qualitatively affect disease gradients or velocity (described below). Lesion count data from four different field experiments (at Hermiston and Madras, OR, in 2002 and 2003; data not shown) were used to provide a range of reasonable values for $b$. When regressions were done on upwind and downwind data to fit the modified power law with $c=$ $0.848 \mathrm{~m}$, estimates of $b$ were $2.29,2.30,2.43,2.52,3.14,3.52$, 4.18, and 5.89. The larger values indicate steeper gradients and were associated with upwind dispersal. Simulations were run using values of $1,2,3,4,5$, and 6 .

Each of the pathogen life cycle components (latent period, infectious period, lesion growth rate, and multiplication rate) was then varied, in turn, to explore its effect on epidemic velocity. Model parameters were chosen to encompass a reasonable range of values for stripe rust on wheat. In some cases, more extreme values were also included.

Latent and infectious periods ranging from 5 to 30 days were used. Latent periods of 11.3 to 23.8 days have been reported for stripe rust on 10 cultivars of spring wheat raised in growth chambers (9). Infectious periods between 6 and 14 days have been observed $(32,57)$. Lesion growth rates were varied from 0 to $100 \%$ per day.

As noted above, DMFR combines the effects of two disease parameters, spore production and infection efficiency. DMFR is directly proportional to each and is therefore also proportional to the product of the two. The product (spore production) $\times$ (infection efficiency) may vary by a factor of up to 100 in cultivars differing in their susceptibility to a pathogen $(30,33)$. In addition to the baseline value of 5 day $^{-1}$, simulations were run with DMFRs of $0.05,0.5$, and $50 \mathrm{day}^{-1}$.

Velocity calculations. In order to calculate the velocity of an isopath at a particular date, the location of the isopath was first estimated. In most cases, the isopath was between two disease assessment locations in the field (or in the simulation output). The estimated location of the isopath was calculated by linear interpolation between these two points. The velocity in meters per week was the distance between this location and the previous week's location. When severity exceeds an isopath level at all points in the plot in the direction of interest, the location of the isopath, and therefore the velocity, cannot be estimated.

\section{RESULTS}

Baseline simulation compared with field epidemic. Disease gradients for both the simulated (power law dispersal) and field epidemics flattened with time (Fig. 2). This flattening is associated with increasing epidemic velocity (Fig. 3, thin and thick solid lines, respectively), and occurred at all levels of disease severity, in both the upwind and downwind directions. Although the shapes of the velocity curves (Fig. 3) were similar, field velocities were smaller than corresponding simulation velocities. Also, disease severity approached $100 \%$ at a slower pace in the field than in the simulation, which can be observed by comparing severities in the focus (Fig. 2).

Comparisons between field and simulated epidemics were made starting at the end of the second latent period, rather than at inoculation (Figs. 2 and 3) because of differences in latent period between the field and the simulations during the early part of the epidemics. The latent period of stripe rust varies with temperature, with long latent periods being associated with cold weather. EPIMUL, on the other hand, uses a constant latent period throughout the simulation. The baseline value for simulation was chosen to match field conditions well during the bulk of the epidemic (approximately weeks 3 through 9 of the 11-week period),

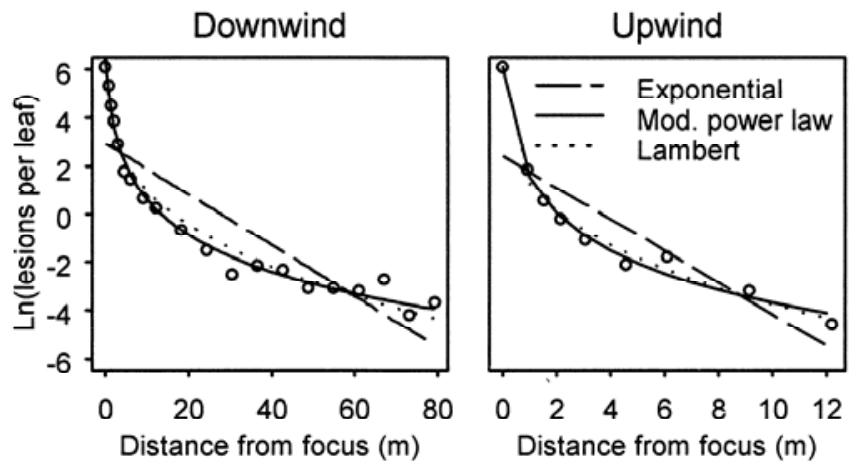

Fig. 1. Three dispersal models fit to primary disease gradients of wheat stripe rust. Open circles are $\log _{\mathrm{e}}$-transformed mean lesion counts from two replicate plots of a field experiment near Hermiston, OR, in 2002. 
but cold weather in the weeks immediately following inoculation in the field delayed the field epidemic relative to the simulation.

Effect of dispersal gradient model. Although the parameters for each gradient model (modified power law, exponential, and Lambert) were estimated using the same field data, the behavior of velocity as a function of time depended strongly on which model was used in the simulation (Fig. 3). Estimates of the shape parameter $n$ for the Lambert model were near 0.3 (Table 1), indicating exponentially unbound tails nearer in shape to the power law than the exponential. Indeed, both the modified power law, which best fit the dispersal data from the field, and the Lambert model resulted in isopath velocities that increased over time. These two models produced similar velocities until the final weeks of the epidemic, when the modified power law velocities exceeded those of the Lambert model. When the exponential was used to determine dispersal in the simulation model, velocities increased for a time, then leveled off. Corroborating this observation, disease gradient plots of these simulations (data not shown) showed that gradients became parallel after the initial buildup period. As expected with parallel disease gradients, the asymptotic velocity of these epidemics did not depend on isopath level. However, the steeper upwind dispersal gradient resulted in a smaller asymptotic velocity, approximately $2 \mathrm{~m} /$ week upwind versus $15 \mathrm{~m} /$ week downwind.

Effects of life cycle components and gradient steepness. Since the qualitative behavior of the isopathic velocity did not appear to depend on severity level in the baseline simulation (Fig. 3 ), a single isopath, the $20 \%$ severity level, was chosen to represent the results of this round of simulations.

The latent period, rate of lesion growth, multiplication rate, and steepness of the dispersal gradient each had a pronounced impact on epidemic velocity (Fig. 4). Increasing the latent period delayed the epidemic, while having little effect on the slope of the velocity curve. Increased lesion growth rate was associated with increased velocity. Decreasing the DMFR reduced velocity by decreasing the slope of the velocity curve. Steeper disease gradients, characterized by larger exponents $(b)$ in the modified power law model, were associated with shallower velocity curves and smaller velocities. The length of the infectious period did not strongly influence the velocity of the epidemic when the infectious period was between 10 and 30 days. Only a very short infectious period, 5 days, was capable of delaying the epidemic.

It is interesting to note that changes in these parameters had no effect on the overall shape of the velocity curve. In every case, velocity increased as the epidemic proceeded.

\section{DISCUSSION}

Conceptually, EPIMUL is a fairly simple model of plant epidemics. It is based on Vanderplankian increase of disease in time (60), it is deterministic, and it takes as constant a number of parameters that in reality fluctuate with factors such as weather and plant maturity. Even so, the model does remarkably well in simulating the behavior of an epidemic as it progresses in time and space. EPIMUL also has the advantage of requiring relatively few input parameters to produce this realistic response, making it possible to apply it to a variety of pathosystems without undue expense.

This version also allows the use of different gradients upwind and downwind, which sets it apart from other models, as does the inclusion of lesion growth. And, although we did not take full advantage of it in the current study, where the simulated plots were long and narrow to match the field plots, EPIMUL allows for simulation of asymmetrical dispersal in two dimensions. The option of using the modified power law, exponential, or Lambert's model for spore dispersal made it possible to confirm the sensitivity of epidemic velocity to dispersal gradient shape, and also to explore the effects of pathogen life cycle components on the
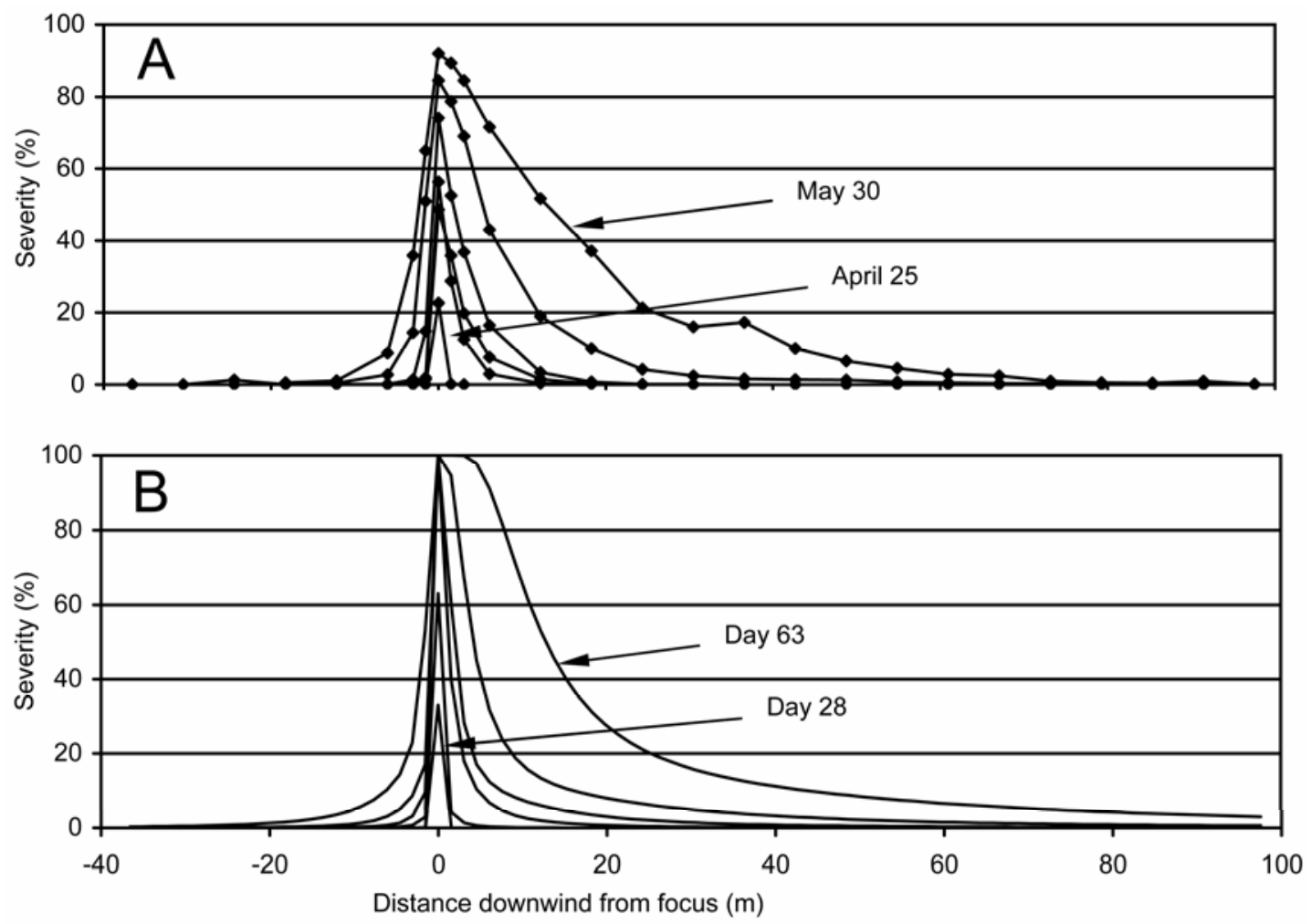

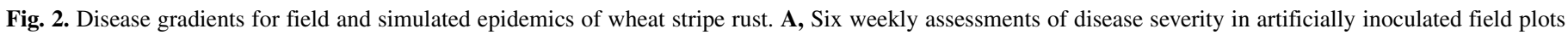

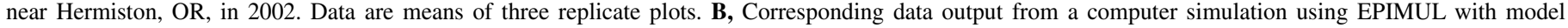

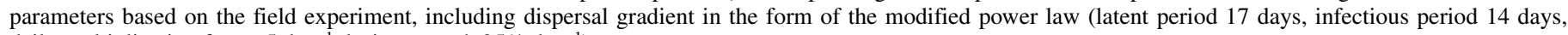
daily multiplication factor 5 day $^{-1}$, lesion growth $25 \%$ day $^{-1}$ ). 
spatiotemporal progress of an epidemic that spreads as a dispersive wave.

EPIMUL successfully re-created the overall characteristics of the Hermiston 2002 field epidemic of wheat stripe rust: greater spread downwind than upwind of the inoculated focus, long-tailed disease gradients that flatten over time, and accelerating movement of isopaths. Early in the epidemics, there was a slight hump in the curves of epidemic velocity versus time for both the field and simulated epidemics, indicating a temporary increase in velocity due to the fact that the pathogen generations are relatively discrete in this stage. Apparently, disease increases in a stepwise fashion initially until the generations begin to overlap.

There were also some differences between the field and simulated epidemics. Upwind of the focus, velocity generally in- creased over time, although the increase in the field seemed to trail off in the last week of observation, while the simulation continued its acceleration. In Oregon, wheat cultivars generally become less susceptible to stripe rust with age, and environmental conditions become less conducive for disease as temperatures rise during late spring and early summer. In the case of slower upwind velocities, these factors likely countered the normal tendency for velocity to continue to increase. In fact, we have noticed a similar phenomenon for a combination of suboptimal environmental conditions and use of cultivar mixtures, which both reduce epidemic velocity (10).

For each severity level, both upwind and downwind, the estimates of velocity according to the EPIMUL simulation were higher than observed in the field. Because of the multiple assump-

\section{Downwind}
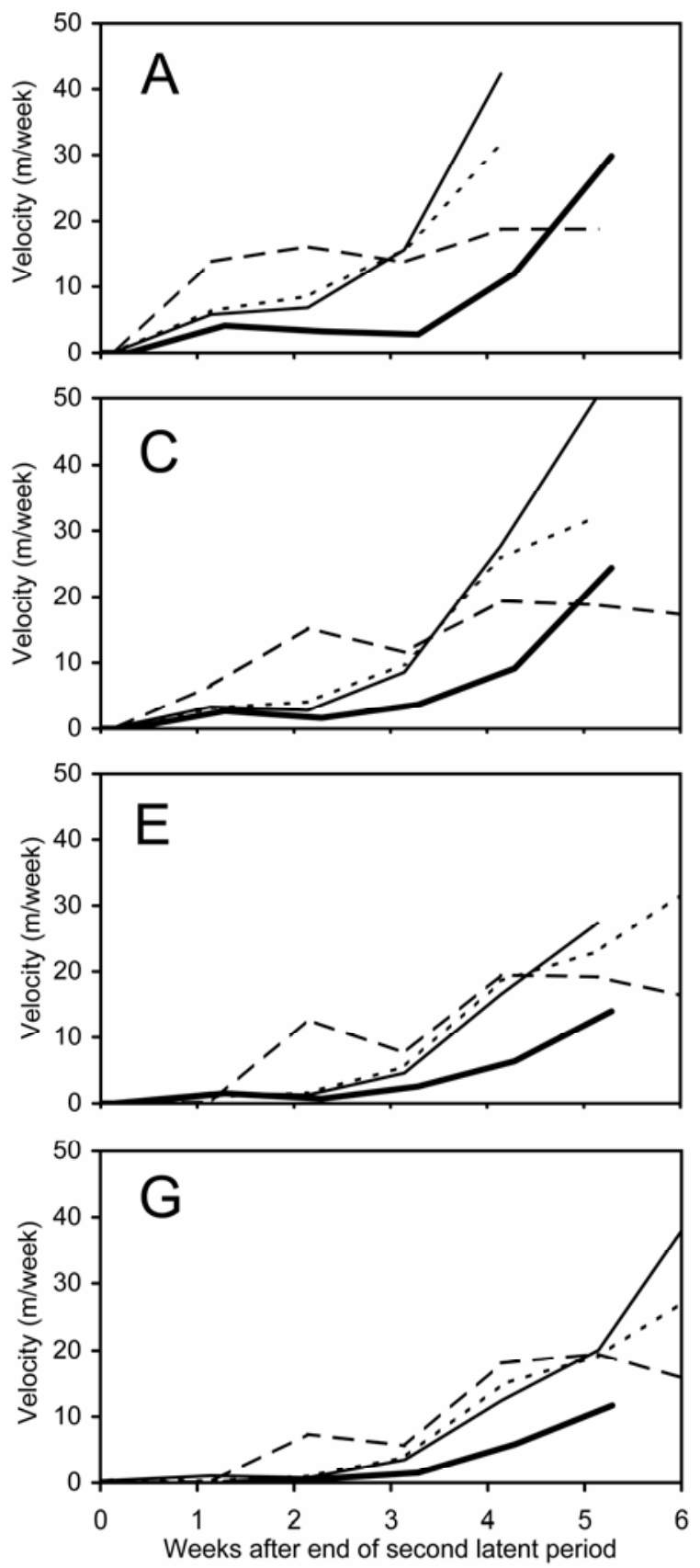

\section{Upwind}
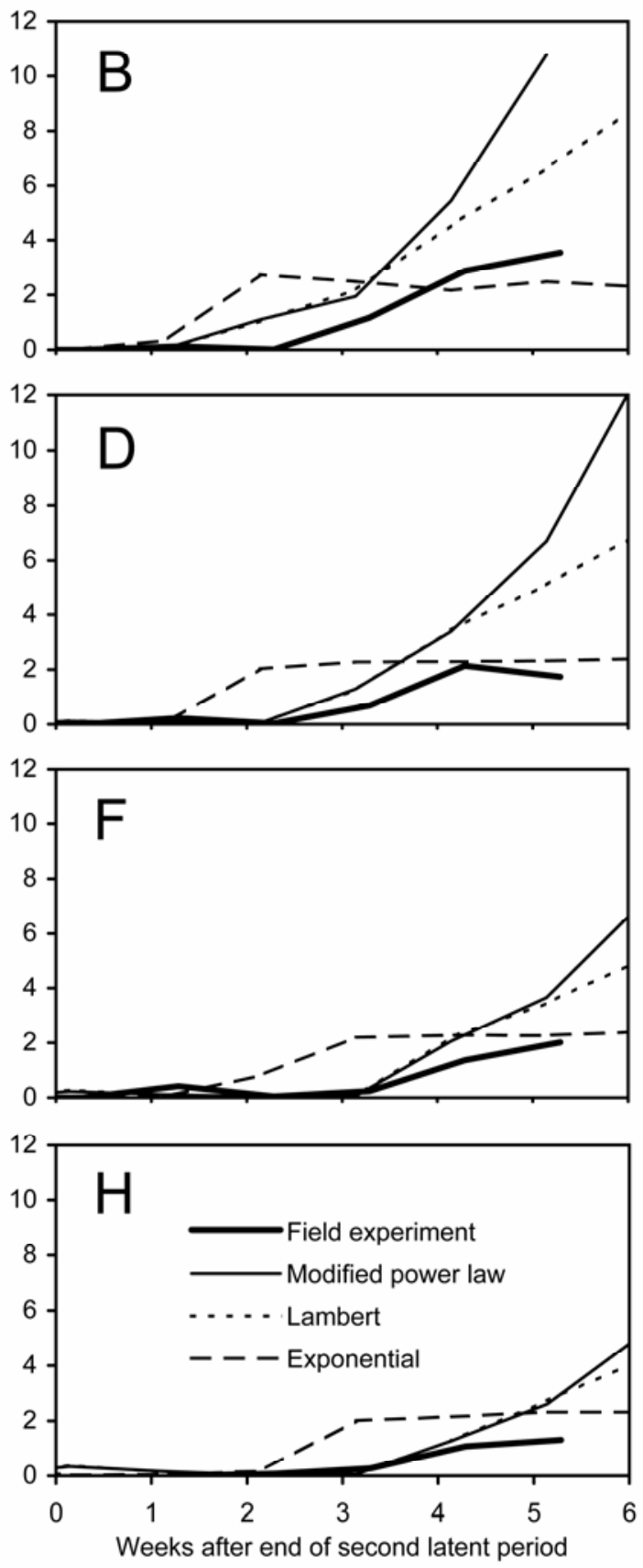

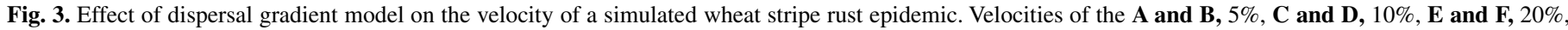

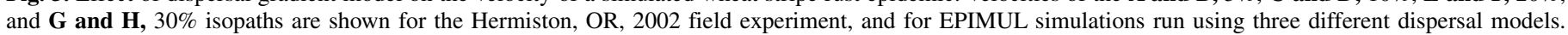
Model parameters were based on the field experiment (latent period 17 days, infectious period 14 days, daily multiplication factor 5 day ${ }^{-1}$, lesion growth $25 \%$ day ${ }^{-1}$ ). 
tions necessary to create a model with a reasonable number of parameters, it is difficult to resolve the sources of such discrepancies. Changes in parameters over time, which the model does not account for, may be involved. Inaccuracies in parameter estimation, particularly DMFR, which is difficult to measure, may contribute. Also, all real-world processes are stochastic at some level (50), while the EPIMUL simulations are deterministic. An epidemic model with deterministic dispersal may produce faster epidemics than the same model with stochastic dispersal $(64,67)$.

The simulated epidemic approached $100 \%$ severity faster than the field epidemic. EPIMUL reduces infection efficiency in direct proportion to the amount of healthy host tissue available for new infections. Apparently, this adjustment is insufficiently restrictive for stripe rust on wheat. The model takes into account only the reduced likelihood that a spore will land on uninfected tissue as disease severity increases. In the field, additional factors may be at work. Plants may develop some physiological resistance to new infections as disease severity increases. Also, stripe rust seems to infect more successfully on lush, green plants (63). The reduced vigor of plants as they become diseased may reduce infection efficiency and slow disease progress relative to the simulated epidemics. This discrepancy may call into question direct compari- son of isopaths at high severity levels (say, 60\% and higher), but again, the actual and simulated epidemics were qualitatively quite similar.

The modified power law, exponential, and Lambert's model were chosen to represent propagule dispersal gradients in this study because of their common use in phytopathology literature, their ability to fit to our field data, and because they have a finite definition at the source $(x=0)$. The latter is a necessary condition for use in simulations so that autoinfection can be quantified. These three models also have different tail shapes, the exponential having an exponentially bound tail, the modified power law having an unbound tail, and the Lambert allowing, via the shape parameter $n$, either bound $(n \geq 1)$ or unbound $(0<n<1)$ tails $(27)$. (When $n=1$, the Lambert model is identical to the exponential.)

A number of other equations have been used to describe dispersal gradients. Gregory's power law, $y=a x^{-b}$ (22), fit our dispersal data well when the focus $(x=0)$ values were excluded from the analysis (45), but was unsuitable for our purposes since it predicts an infinite value of $y$ at $x=0$. Variations on the modified power law (equation 1) include the Pareto probability distribution, $y=$ $\alpha \theta^{\alpha}(x+\theta)^{-(\alpha+1)}(36)$, and the Cauchy distribution, $y=(2 / \pi b) 1 /[1+$ $\left.(x / b)^{2}\right](51)$, among others (20). However, these are not necessar-
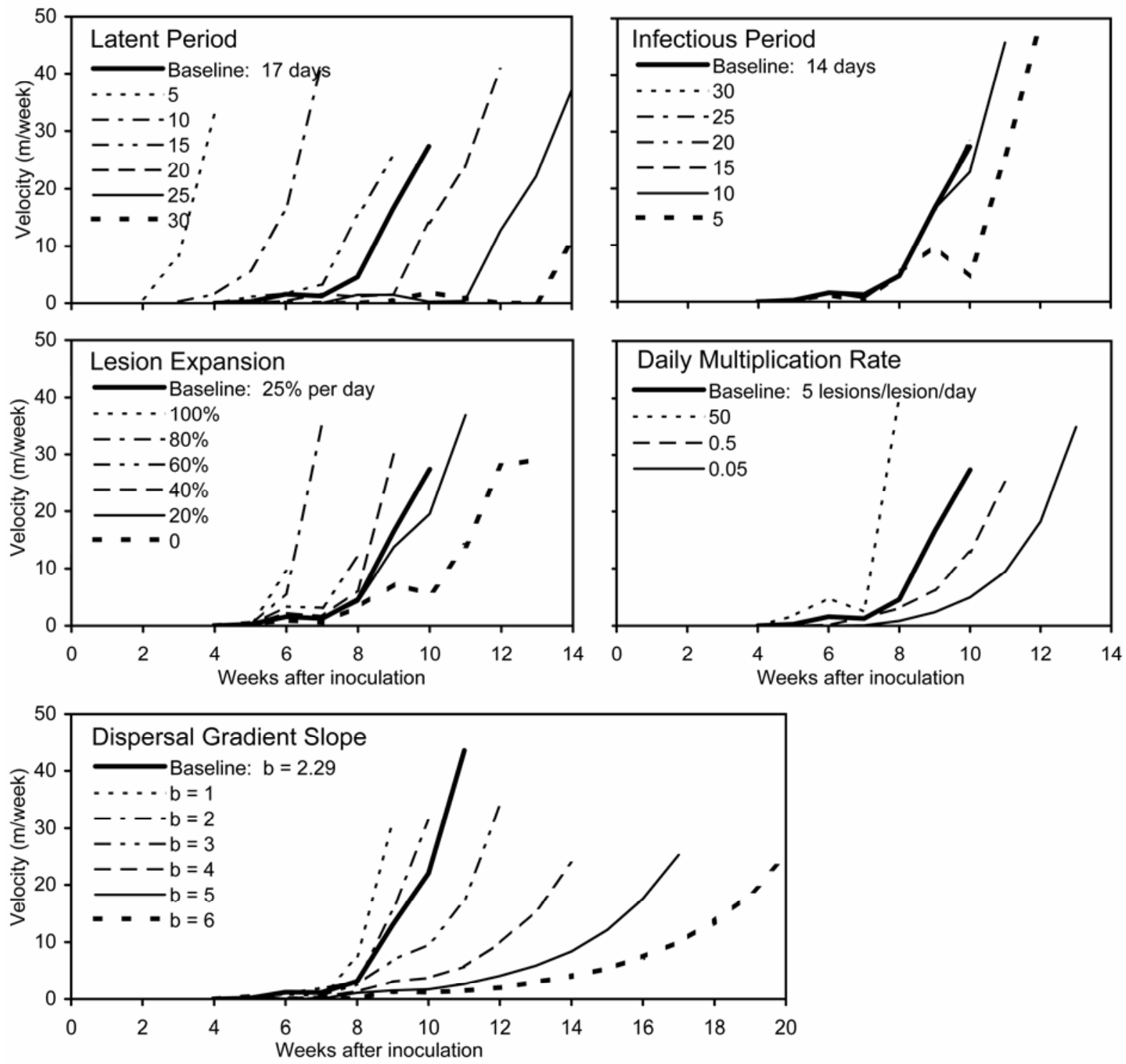

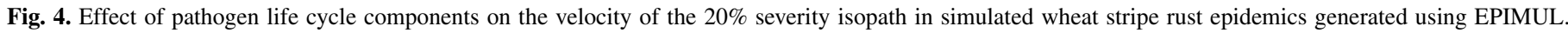

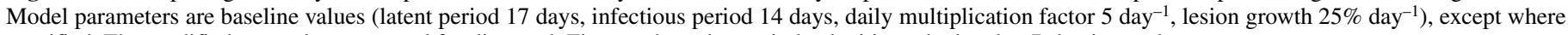
specified. The modified power law was used for dispersal. Figures show downwind velocities calculated at 7-day intervals. 
ily equivalent, and neither the Pareto nor the Cauchy fit our field data well. The Cauchy, for instance, followed the shape of the gradients fairly well at distances $\geq 10 \mathrm{~m}$, but underestimated dispersal nearer the source (data not shown). Depending on the choice of $n$, the Lambert can be equivalent to other equations that have been used to describe gradients. For example, the root function, $y=1 / 4 a^{2} \exp \left(-a x^{-1 / 2}\right)(20)$, results when $n=1 / 2$; a normal curve results when $n=2(27)$.

Our simulations showed how sensitive the velocity of an epidemic is to the pattern of spore dispersal. In particular, the tail of the dispersal gradient-the spores that travel far from their source-drives the behavior of the epidemic. Epidemics simulated using either the Lambert or the modified power law model had velocities that increased over time, with no apparent tendency to level off. The difference in the shape of the modified power law and Lambert gradients, though subtle (Fig. 1), did cause consistent differences in the velocity (Fig. 3). The modified power law velocity ran just under the Lambert velocity until the last few weeks of the epidemic, when it crossed over and overtook the Lambert. With exponential dispersal, velocity increased at the beginning of the epidemic, then leveled off. This result is in agreement with the findings of other simulation and analytical studies $(14,15,39,50)$, and confirms the utility of EPIMUL's simple model.

These results confirm that characterizing the dispersal gradient is central to understanding how a focal epidemic progresses in time and space. In the dispersal of $P$. striiformis spores in the windy conditions of eastern Oregon, the modified power law provided a clearly better fit to the data than did the exponential model. In determining this, we had the advantage of large plots and environmental conditions favorable to disease. The exponential and power law models tend to fit equally well close to the source $(4,45)$. In fact, had dispersal data been collected only to $6.1 \mathrm{~m}$ downwind of the focus - farther than many gradient studies - the two models would have been virtually indistinguishable $\left(R^{2}=0.939\right.$ for the exponential and $R^{2}=0.992$ for the modified power law, with seven data points). It became clearer as we moved outward from the focus that the exponential model was inappropriate and underestimated dispersal at large distances as well as close to the source. Because conditions were favorable to infection, the plants in the focus provided an abundant source of inoculum, and during the first generation of dispersal, non-zero levels of disease were detected relatively far from the focus (79.2 $\mathrm{m}$ downwind, $12.2 \mathrm{~m}$ upwind). Other disease systems may require different scales of observation to characterize dispersal.

Having observed the acceleration of this epidemic in the field and determined that it can be explained via simulations using a power law dispersal model, the next logical question is whether this acceleration can continue over larger spans of time and space. At present, this is unknown. The dispersal gradient may or may not continue to follow the power law on larger scales. Continued acceleration of epidemics may be hindered by geographic factors, climatological factors, and decreasing availability of suscepts farther from the source $(5,23)$, as well as seasonal changes in weather and host availability.

Our findings that latent period, multiplication rate, and dispersal gradient steepness had strong effects on epidemic velocity are consistent with the results of the analytic studies of van den Bosch et al. (59) and Minogue and Fry (37), although their work was based on epidemics that expanded at constant velocities. Minogue and Fry (37) concluded, as we did, that infectious period had little effect on velocity, except when the infectious period was very small. However, with the exception of Ferrandino's analytic study (15), which examined the relationship between multiplication rate and velocity, the specific effects of pathogen life cycle components on epidemics expanding as dispersive waves had not been investigated. Our simulations showed a qualitative difference between the effects of multiplication rate and gradient slope on one hand, and latent period on the other. Lengthening the latent period delayed the epidemics, but had no effect on the rate of increase of the velocity. Steeper gradients and smaller multiplication rates, in contrast, slowed epidemics by slowing the rate of increase of velocity. This relationship between decreasing multiplication rate and slower velocity increase agrees with Ferrandino's work (15). Lesion growth also had a strong, though more complex, impact on velocity. Reducing lesion growth may delay the epidemic, slow the rate of velocity increase, or both. These components of the pathogen's life cycle have been shown in other studies to have similar effects on nonspatial measures of disease, such as AUDPC $(33,43)$, disease severity over time $(52,54,61)$, and $r(29)$.

The present study has the advantage of combining dispersal gradient data derived directly from the field, subsequent estimates of epidemic velocity derived from those same field plots, and a spatially explicit simulation model that reliably reproduced the dynamics of the field epidemics. Our study confirms that the shape of the dispersal function is key to the nature of epidemic velocity. In our simulations, a traveling wave of constant velocity was attained, after a period of stabilization, only for an exponential model. However, non-exponentially bound dispersal models provided better fits to primary disease gradient data of wheat stripe rust and always resulted in dispersive epidemic waves when incorporated into simulations. Pathogen life cycle components had quantitative effects on epidemic velocity, and were usually similar to those previously reported for epidemic progression in time and expansion of traveling wave epidemics. Life cycle components, however, did not influence velocity qualitatively when power law dispersal was simulated: velocity always increased in time over the wide range of input values for life cycle components that we investigated. Thus, dispersive epidemic waves could result for a diversity of plant diseases, provided that their dispersal follows a non-exponentially bound function such as the power law.

\section{LITERATURE CITED}

1. Alderman, S. C., Nutter, F. W., and Labrinos, J. L. 1989. Spatial and temporal analysis of spread of late leaf spot of peanut. Phytopathology 79:837-844.

2. Andow, D. A., Kareiva, P. M., Levin, S. A., and Okubo, A. 1990. Spread of invading organisms. Landsc. Ecol. 4:177-188.

3. Assefa, H., van den Bosch, F., and Zadoks, J. C. 1995. Focus expansion of bean rust in cultivar mixtures. Plant Pathol. 44:503-509.

4. Aylor, D. E. 1987. Deposition gradients of urediniospores of Puccinia recondita near a source. Phytopathology 77:1442-1448.

5. Aylor, D. E. 1999. Biophysical scaling and the passive dispersal of fungus spores: Relationship to integrated pest management strategies. Agric. For. Meteorol. 97:275-292.

6. Aylor, D. E., and Ferrandino, F. J. 1989. Temporal and spatial development of bean rust epidemics initiated from an inoculated line source. Phytopathology 79:146-151.

7. Berger, R. D., Bergamin Filho, A., and Amorim, L. 1997. Lesion expansion as an epidemic component. Phytopathology 87:1005-1013.

8. Berger, R. D., and Luke, H. H. 1979. Spatial and temporal spread of oat crown rust. Phytopathology 69:1199-1201.

9. Broers, L. H. M. 1997. Components of quantitative resistance to yellow rust in ten spring bread wheat cultivars and their relations with field assessments. Euphytica 96:215-233.

10. Cowger, C., Wallace, L. D., and Mundt, C. C. 2005. Velocity of spread of wheat stripe rust epidemics. Phytopathology 95:972-982.

11. Díaz-Lago, J. E., Stuthman, D. D., and Leonard, K. J. 2003. Evaluation of components of partial resistance to oat crown rust using digital image analysis. Plant Dis. 87:667-674.

12. Dowkiw, A., Husson, C., Frey, P., Pinon, J., and Bastien, C. 2003. Partial resistance to Melampsora larici-populina leaf rust in hybrid poplars: Genetic variability in inoculated excised leaf disk bioassay and relationship with complete resistance. Phytopathology 93:421-427.

13. Emge, R. G., Kingsolver, C. H., and Johnson, D. R. 1975. Growth of the sporulating zone of Puccinia striiformis and its relationship to stripe rust epiphytology. Phytopathology 65:679-681.

14. Ferrandino, F. J. 1989. Spatial and temporal variation of a defoliating plant disease and reduction in yield. Agric. For. Meteorol. 47:273-289. 
15. Ferrandino, F. J. 1993. Dispersive epidemic waves: I. Focus expansion within a linear planting. Phytopathology 83:795-802.

16. Ferrandino, F. J. 1996. Length scale of disease spread: Fact or artifact of experimental geometry. Phytopathology 86:806-811.

17. Fitt, B. D. L., Gregory, P. H., Todd, A. D., McCartney, H. A., and Macdonald, O. C. 1987. Spore dispersal and plant disease gradients; A comparison between two empirical models. J. Phytopathol. 118:227-242.

18. Fitt, B. D. L., and McCartney, H. A. 1986. Spore dispersal in relation to epidemic models. Pages 311-345 in: Plant Disease Epidemiology, Vol. 1. K. J. Leonard and W. E. Fry, eds. Macmillan, New York.

19. Frantzen, J., and Müller-Schärer, H. 1998. A theory relating focal epidemics to crop-weed interactions. Phytopathology 88:180-184.

20. Frantzen, J., and van den Bosch, F. 2000. Spread of organisms: Can traveling and dispersive waves be distinguished? Basic Appl. Ecol. 1:83-91.

21. Gregory, P. H. 1948. The multiple infection transformation. Ann. Appl. Biol. 35:412-417.

22. Gregory, P. H. 1968. Interpreting plant disease dispersal gradients. Annu. Rev. Phytopathol. 6:189-212.

23. Heesterbeek, J. A. P., and Zadoks, J. C. 1987. Modelling pandemics of quarantine pests and diseases: Problems and perspectives. Crop Prot. 6:211-221.

24. Jeger, M. J., Jones, D. G., and Griffiths, E. 1983. Disease spread of nonspecialized fungal pathogens from inoculated point sources in intraspecific mixed stands of cereal cultivars. Ann. Appl. Biol. 102:237-244.

25. Kampmeijer, P., and Zadoks, J. C. 1977. EPIMUL, A Simulator of Foci and Epidemics in Mixtures of Resistant and Susceptible Plants, Mosaics and Multilines. Centre for Agricultural Publishing and Documentation, Wageningen, the Netherlands.

26. Kiyosawa, S., and Shiyomi, M. 1972. A theoretical evaluation of mixing resistant variety with susceptible variety for controlling plant diseases. Ann. Phytopathol. Soc. Jpn. 38:41-51.

27. Lambert, D. H., Villareal, R. L., and Mackenzie, D. R. 1980. A general model for gradient analysis. Phytopathol. Z. 98:150-154.

28. Lannou, C., de Vallavieille-Pope, C., and Goyeau, H. 1994. Host mixture efficacy in disease control: Effect of lesion growth analysed through computer-simulated epidemics. Plant Pathol. 43:651-662.

29. Leonard, K. J., and Mundt, C. C. 1984. Methods for estimating epidemiological effects of quantitative resistance to plant diseases. Theor. Appl. Genet. 67:219-230.

30. Loughman, R., Wilson, R. E., and Thomas, G. J. 1996. Components of resistance to Mycosphaerella graminicola and Phaeosphaeria nodorum in spring wheats. Euphytica 89:377-385.

31. Luo, Y., and TeBeest, D. O. 1997. Infection components of wild-type and mutant strains of Colletotrichum gloeosporioides $\mathrm{f}$. sp. aeschynomene on northern jointvetch. Plant Dis. 81:404-409.

32. Luo, Y., and Zeng, S. 1988. Component analysis of slow-rusting resistance of wheat cultivars to stripe rust (Puccinia striiformis) (I). Sci. Sin. Ser. B (Chem. Biol. Agric. Med. Earth Sci.) 31:217-227.

33. Luo, Y., and Zeng, S. M. 1995. Simulation studies on epidemics of wheat stripe rust (Puccinia striiformis) on slow-rusting cultivars and analysis of effects of resistance components. Plant Pathol. 44:340-349.

34. Madden, L. V., Knoke, J. K., and Louie, R. 1990. Spread of maize chlorotic dwarf virus in maize fields by its leafhopper vector, Graminella nigrifrons. Phytopathology 80:291-298.

35. Minogue, K. P. 1986. Disease gradients and the spread of disease. Pages 285-310 in: Plant Disease Epidemiology Vol. 1. K. J. Leonard and W. E. Fry, eds. Macmillan, New York.

36. Minogue, K. P. 1989. Diffusion and spatial probability models for disease spread. Pages 127-143 in: Spatial Components of Plant Disease Epidemics. M. J. Jeger, ed. Prentice Hall, Englewood Cliffs, NJ.

37. Minogue, K. P., and Fry, W. E. 1983. Models for the spread of disease: Model description. Phytopathology 73:1168-1173.

38. Minogue, K. P., and Fry, W. E. 1983. Models for the spread of plant disease: Some experimental results. Phytopathology 73:1173-1176.

39. Mollison, D. 1977. Spatial contact models for ecological and epidemic spread. J. R. Statist. Soc. B 39:283-326.

40. Mundt, C. C., and Leonard, K. J. 1985. A modification of Gregory's model for describing plant disease gradients. Phytopathology 75:930-935.

41. Mundt, C. C., Leonard, K. J., Thal, W. M., and Fulton, J. H. 1986. Computerized simulation of crown rust epidemics in mixtures of immune and susceptible oat plants with different genotype unit areas and spatial distributions of initial disease. Phytopathology 76:590-598.

42. Parlevliet, J. E. 1979. Components of resistance that reduce the rate of epidemic development. Annu. Rev. Phytopathol. 17:203-232.

43. Rossi, V., Giosuè, S., and Racca, P. 1999. A model integrating components of rate-reducing resistance to Cercospora leaf spot in sugar beet. J. Phytopathol. 147:339-346.

44. Sache, I., and Zadoks, J. C. 1996. Spread of faba bean rust over a discontinuous field. Eur. J. Plant Pathol. 102:51-60.

45. Sackett, K. E., and Mundt, C. C. 2005. Primary disease gradients of wheat stripe rust in large field plots. Phytopathology 95:983-991.

46. Seebold, K. W., Kucharek, T. A., Datnoff, L. E., Correa-Victoria, F. J., and Marchetti, M. A. 2001. The influence of silicon on components of resistance to blast in susceptible, partially resistant, and resistant cultivars of rice. Phytopathology 91:63-69.

47. Shaner, G., and Hess, F. D. 1978. Equations for integrating components of slow leaf-rusting resistance in wheat. Phytopathology 68:1464-1469.

48. Shaner, G., and Powelson, R. C. 1971. Epidemiology of stripe rust of wheat, 1961-1968. Oregon Agric. Exp. Stn. Bull. 117.

49. Shaw, M. W. 1983. Rapid movement of a B-chromosome frequency cline in Myrmeleotettix maculatus (Orthoptera: Acrididae). Heredity 50:1-14.

50. Shaw, M. W. 1994. Modeling stochastic processes in plant pathology. Annu. Rev. Phytopathol. 32:523-544.

51. Shaw, M. W. 1995. Simulation of population expansion and spatial pattern when individual dispersal distributions do not decline exponentially with distance. Proc. R. Soc. Lond. B Biol. Sci. 259:243-248.

52. Shrum, R. 1975. Simulation of wheat stripe rust (Puccinia striiformis West.) using EPIDEMIC, a flexible plant disease simulator. Prog. Rep. Pa. Agric. Exp. Stn. 347

53. Sillero, J. C., Moreno, M. T., and Rubiales, D. 2000. Characterization of new sources of resistance to Uromyces viciae-fabae in a germplasm collection of Vicia faba. Plant Pathol. 49:389-395.

54. Teng, P. S., Blackie, M. J., and Close, R. C. 1977. A simulation analysis of crop yield loss due to rust disease. Agric. Syst. 2:189-198.

55. Tollenaar, H., and Houston, B. R. 1967. A study on the epidemiology of stripe rust, Puccinia striiformis West., in California. Can. J. Bot. 24:291307.

56. Tredway, L. P., Stevenson, K. L., and Burpee, L. L. 2003. Components of resistance to Magnaporthe grisea in 'Coyote' and 'Coronado' tall fescue. Plant Dis. 87:906-912.

57. van den Bosch, F., Frinking, H. D., Metz, J. A. J., and Zadoks, J. C. 1988. Focus expansion in plant disease. III: Two experimental examples. Phytopathology 78:919-925.

58. van den Bosch, F., Zadoks, J. C., and Metz, J. A. J. 1988. Focus expansion in plant disease. I: The constant rate of focus expansion. Phytopathology 78:54-58.

59. van den Bosch, F., Zadoks, J. C., and Metz, J. A. J. 1988. Focus expansion in plant disease. II: Realistic parameter-sparse models. Phytopathology 78:59-64.

60. Vanderplank, J. E. 1963. Plant Diseases: Epidemics and Control. Academic Press, New York.

61. van Oijen, M. 1992. Selection and use of a mathematical model to evaluate components of resistance to Phytophthora infestans in potato. Neth. J. Plant Pathol. 98:192-202.

62. Waggoner, P. E., and Aylor, D. E. 2000. Epidemiology: A science of patterns. Annu. Rev. Phytopathol. 38:71-94.

63. Zadoks, J. C. 1961. Yellow rust on wheat, studies in epidemiology and physiologic specialization. Tijdschr. Plantenziekten 67:69-256.

64. Zadoks, J. C. 2000. Foci, small and large: A specific class of biological invasion. Pages 292-317 in: The Geometry of Ecological Interactions: Simplifying Spatial Complexity. U. Dieckmann, R. Law, and J. A. J. Metz, eds. Cambridge University Press, Cambridge.

65. Zadoks, J. C. 2001. Plant disease epidemiology in the twentieth century: A picture by means of selected controversies. Plant Dis. 85:808-815.

66. Zadoks, J. C., and Kampmeijer, P. 1977. The role of crop populations and their deployment, illustrated by means of a simulator, EPIMUL76. Ann. N.Y. Acad. Sci. 287:164-190.

67. Zadoks, J. C., and van den Bosch, F. 1994. On the spread of plant disease: A theory on foci. Annu. Rev. Phytopathol. 32:503-521.

68. Zawolek, M. W., and Zadoks, J. C. 1992. Studies in focus development: An optimum for the dual dispersal of plant pathogens. Phytopathology $82: 1288-1297$ 